\title{
A utilização da monitorização ambulatória da pressão arterial em cuidados de saúde primários
}

Ana Cristina B. Nobre, ${ }^{*}$ Sandra Maturino Santos, ${ }^{* *}$ Inês Pinheiro da Silva, ${ }^{* * *}$ Rita Rodrigues Soares ${ }^{* * *}$

\section{RESUMO}

Introdução: A medição ocasional da pressão arterial tem sido utilizada como método diagnóstico da hipertensão arterial para estabelecer o prognóstico do doente e para avaliar a eficácia do tratamento anti-hipertensivo instituído.

A monitorização ambulatória da pressão arterial é um meio auxiliar de diagnóstico importante na avaliação e seguimento do doente hipertenso.

Objectivo: Apresentar uma revisão actualizada das recomendações para a sua utilização, nomeadamente em cuidados de saúde primários.

Métodos: Foi efectuada uma pesquisa bibliográfica de artigos de revisão, na base de dados Medline, nos últimos 8 anos, em língua inglesa e espanhola, através da utilização das palavras-chave «ambulatory blood pressure» e «arterial hypertension» .

Conclusões: Este exame auxiliar de diagnóstico, tem um valor preditivo para eventos cardiovasculares maior que a pressão arterial avaliada no consultório. A sua realização pode melhorar a avaliação do risco cardiovascular dos doentes hipertensos. As principais recomendações para a sua utilização são: a abordagem da hipertensão refractária, os doentes com eventos cardiovasculares prévios ou com lesões de órgãos alvo, o diagnóstico de hipertensão da bata branca e de hipertensão mascarada, a identificação do ritmo circadiano anormal da pressão arterial, mais frequente nos idosos e responsável pelo agravamento do seu prognóstico, a avaliação dos doentes diabéticos e das grávidas com valores de pressão arterial elevados.

Palavras-chave: Hipertensão; Hipertensão da Bata Branca; Hipertensão Mascarada; MAPA; Pressão Arterial Avaliada no Consultório; Cuidados de Saúde Primários; Doença Cardiovascular.

\section{INTRODUÇÃO}

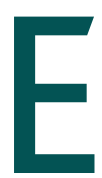

ntre outras competências, o Médico de Família tem que saber gerir os problemas de saúde dos seus pacientes e lidar com esses mesmos problemas em todas as suas dimensões, nomeadamente física, psicológica, social, cultural e existencial. A hipertensão arterial (HTA) é um deles.

O principal objectivo do tratamento do doente hipertenso é obter a longo prazo a máxima redução da morbilidade e mortalidade cardiovascular e renal. Tal poderá ser conseguido pela redução dos valores elevados

*Assistente de Clínica Geral, Centro de Saúde de Santarém

Unidade de Saúde Familiar de S. Domingos

** Médica Interna de Medicina Geral e Familiar na USF de S. Domingos

*** Médica Interna de Medicina Geral e Familiar na USF de S. Domingos da pressão arterial e pelo tratamento dos factores de risco modificáveis e de doenças associadas.

A variação contínua dos valores da pressão arterial, quer diastólicos quer sistólicos, e a correlação directa entre a progressão destes valores e o aumento de risco de doença cérebro e cardiovascular, torna evidente a importância de um adequado controlo da pressão arterial.

Devido à existência de mecanismos de adaptação, a pressão arterial varia ao longo das 24 horas. O valor registado em consulta pode não ser representativo dos níveis reais de um indivíduo.

A monitorização ambulatória da pressão arterial (MAPA), permite-nos eliminar a potencial discrepância entre a pressão arterial do consultório e o perfil dinâ- 
mico da pressão arterial no quotidiano do paciente.

O recurso a este exame auxiliar de diagnóstico deve ser considerado na prática clínica, salvaguardando as suas indicações principais. ${ }^{1}$

\section{OBJECTIVO}

Apresentar uma revisão actualizada das recomendações para a utilização da MAPA, nomeadamente em cuidados de saúde primários.

\section{MÉTODOS}

Foi efectuada uma pesquisa bibliográfica de artigos de revisão, na base de dados Medline, nos últimos 8 anos, em língua inglesa e espanhola, através da utilização das palavras-chave «ambulatory blood pressure» e «arterial hypertension».

Foram seleccionados os artigos mais recentes e simultaneamente os que se consideraram mais pertinentes.

\section{A UTILIZAÇÂO DA MONITORIZAÇÃO AMBULATÓRIA DA PRESSÃO ARTERIAL}

Trata-se de um importante auxiliar para o diagnóstico e para o seguimento clínico dos doentes com HTA, com menor erro que as avaliações efectuadas em consulta. ${ }^{1,2}$

A MAPA é o melhor factor preditivo dos eventos cardiovasculares e da mortalidade para a HTA do que a pressão arterial avaliada no consultório, tanto na população em geral como nos grupos de alto risco. ${ }^{3}$

Devido ao seu custo e ao incómodo que causa aos doentes, o seu uso deve ser reservado (grau de recomendação B, baseada em estudos consistentes ${ }^{1}$ para situações como: ${ }^{1-5}$

- a abordagem da hipertensão refractária;

- o diagnóstico da hipertensão da bata branca;

- o diagnóstico da hipertensão mascarada;

- a avaliação de doentes com eventos cardiovasculares prévios ou com lesões de órgãos alvo;

- a avaliação da resposta ao tratamento anti-hipertensor, identificando os doentes com bom ou mau controlo e os períodos de insuficiente cobertura terapêutica;

- a detecção de episódios de hipotensão relacionados com o tratamento farmacológico;

- a avaliação do idoso (idade igual ou superior a 65 anos);

- a avaliação do doente diabético com HTA, para quem as exigências de controlo são ainda maiores que na população em geral;

- a identificação da hipertensão nocturna e,

- o diagnóstico e tratamento das consequências da HTA durante a gravidez. ${ }^{5}$

\section{A MONITORIZAÇÃO AMBULATÓRIA DA PRESSÃO ARTERIAL EM CUIDADOS DE SAÚDE PRIMÁRIOS} Hipertensão da bata branca

Definida como uma pressão arterial persistentemente elevada, em ambiente de consultório ou hospitalar, enquanto fora destes locais os valores são normais.

Tem sido reconhecida em diversos estudos internacionais a importância do efeito da bata branca no diagnóstico e controlo da HTA.

Não chega utilizar apenas os valores tensionais obtidos da forma tradicional para se tomar a decisão de instituir terapêutica anti-hipertensora. Parece haver evidências que os indivíduos atrás referidos apresentam um risco cardiovascular intermédio entre os doentes hipertensos e os normotensos.

Os doentes com hipertensão da bata branca necessitam de uma maior vigilância, de alteração dos estilos de vida e eventualmente de terapêutica farmacológica. ${ }^{3-5}$

Os idosos apresentam uma maior variabilidade na pressão arterial e uma maior tendência para o efeito da bata branca, com uma prevalência de $17 \%{ }^{4}$

\section{Hipertensão mascarada}

A hipertensão mascarada ocorre quando a pressão arterial ambulatória se encontra elevada, mas a pressão avaliada no consultório é normal. Foi observada em 9 a 13\% da população em geral e apenas foi reconhecida com o aparecimento da MAPA. ${ }^{4}$

É mais frequente nos grupos etários mais jovens sendo difícil de detectar.

Suspeita-se desta forma de hipertensão em indivíduos com hábitos tabágicos, com sedentarismo, com diabetes, em doentes com múltiplos factores de risco cardiovascular e nos que têm antecedentes familiares de HTA nos dois pais. ${ }^{5}$

Alguns estudos apontam para a existência de maior número de lesões de órgãos-alvo e de eventos cardiovasculares neste grupo de doentes, quando comparados com os normotensos e lesões semelhantes quando 
comparados com os do grupo de hipertensos. ${ }^{4}$

Por este motivo, é importante tratar estes indivíduos, pois têm um risco 2,5 vezes superior de morte cardiovascular ou de acidente vascular cerebral (AVC) do que os normotensos. ${ }^{4}$

\section{Seguimento de doentes hipertensos}

No seguimento de doentes hipertensos há consenso e evidência sobre uma melhor correlação entre lesões em órgãos alvo e os valores da pressão arterial medidos em MAPA, comparando com as medições casuais. ${ }^{1,2,6} \mathrm{~A}$ MAPA tem maior relação com a hipertrofia ventricular esquerda, com a aterosclerose carotídea e com a micro-albuminúria do que a pressão arterial avaliada no consultório. Esta superioridade pode ser potenciada nos idosos. $^{4,7}$

\section{A avaliação do ritmo circadiano da pressão arterial}

A MAPA permite a avaliação não invasiva da pressão arterial durante as 24 horas, a intervalos curtos, no ambiente natural do indivíduo e durante a execução das suas actividades habituais. Observam-se níveis tensionais mais elevados durante as horas em vigília e menores durante o sono, tanto em normotensos como em hipertensos.

A avaliação do ritmo circadiano da pressão arterial permite identificar os indivíduos que não apresentam o padrão de dipping nocturno (redução de 10 a 20\% da pressão arterial em relação ao valor diurno), ou seja, os não-dippers.

Estes indivíduos têm um aumento da incidência das lesões de órgãos-alvo e do risco cardiovascular, independentemente da média dos valores da pressão arterial nas 24 horas. ${ }^{4,5} \mathrm{O}$ não dipping também permite predizer uma lesão renal progressiva nos doentes diabéticos e hipertensos.

\section{HTA durante a gravidez}

A HTA na gravidez representa um risco acrescido para a mulher e para o bebé.

A MAPA pode fazer suspeitar da pré-eclâmpsia na grávida. Esta avaliação tem melhor correlação com a proteinúria que a medição convencional e é um bom indicador das complicações da HTA na gravidez.

Além disso, a hipertensão da bata branca pode ocorrer em $30 \%$ das grávidas e nesse caso são medicadas com antihipertensores e referenciadas aos cuidados de saúde secundários desnecessariamente. Também tendem a ter mais cesarianas que as normotensas. ${ }^{5,8}$

Se este exame for solicitado nestas grávidas, evitarse-ia o início de terapêutica antihipertensiva, desnecessária na existência da hipertensão de bata branca.

\section{CONCLUSÕES}

A MAPA fornece informação diagnóstica e prognóstica superior à da pressão arterial avaliada no consultório. Isto torna-se mais evidente nos idosos que apresentam uma maior variabilidade na pressão arterial e uma maior tendência para um efeito da bata branca.

A avaliação do efeito da medicação instituída, o conhecimento do perfil tensional do nosso doente e o aumento do número de diabéticos e de idosos nos nossos ficheiros clínicos, poderão justificar a utilização deste método na nossa prática diária.

Embora a MAPA seja dispendiosa, em comparação com outros métodos de avaliação da pressão arterial, os resultados que permite obter contribuem para a redução dos custos com a saúde. ${ }^{9}$

A utilização parcimoniosa da MAPA, fundamentada em conclusões cientificamente aceitáveis e a ampliação do seu uso, dar-nos-ão os benefícios necessários à utilização plena do método, explorando-lhe os máximos benefícios a favor da compreensão da HTA e dos cuidados necessários para o seu tratamento.

\section{REFERÊNCIAS BIBLIOGRÁFICAS}

1. Myers MG. Ambulatory blood pressure monitoring for routine clinical practice. Hypertension 2005 Apr; 45 (4): 483-4.

2. Stephens MB, DeGruy F. Does ambulatory blood pressure monitoring aid in the management of patients with hypertension? The Journal of Family Practice 2002 Jan; 51 (1): 15.

3. Verdecchia P, Angeli F, Gattobigio R. Clinical usefulness of ambulatory blood pressure monitoring. J Am Soc Nephrol 2004 Jan; 15 Suppl 1: S30-S33.

4. Ommen ES, Lipkowitz MS. The role of ambulatory BP monitoring in clinical care. Geriatrics 2007 Aug; 62 (8): 11-4.

5. $\mathrm{O}$ `Brien E.Ambulatory blood pressure monitoring in the management of hypertension. Heart 2003 May; 89 (5): 571-6.

6. Mc-Nab P, Jalil JE. Valor pronóstico de la monitoría ambulatória de presión arterial en pacientes hipertensos: observaciones en la literatura. Rev Med Chil 2005 Jan; 133 (1): 89-95.

7. Staessen JA, Thijs L, Fagard R, O'Brien ET, Clement D, Leeuw PW, et al. Predicting cardiovascular risk using conventional vs ambulatory blood pressure in older patients with systolic hypertension. JAMA 1999 Aug 11; 282 (6): 539-46. 
8. O `Brien E, Coats A, Owens P, Petrie J, Padfield PL, Littler WA, et al. Use and interpretation of ambulatory blood pressure monitoring: recommendations of the British Hypertension Society. BMJ 2000 Apr 22; 320 (7242): 1128-34.

9. Pickering TG, Shimbo D, Haas D. Ambulatory blood-pressure monitoring. N Engl J Med 2006 Jun 1; 354 (22): 2368-74.

\section{ABSTRACT}

Introduction: Occasional blood pressure measurement has been used as a diagnostic method in hypertension, in order to assess the patient prognostic and evaluate the efficiency of the applied anti-hypertensive treatment.

Ambulatory blood pressure monitoring is an auxiliary means of diagnostic of importance in the evaluation and follow-up of the hypertensive patient

Goal: To present an updated review of the recommendations for its use, namely in the context of primary healthcare.

Methods: A bibliographical research was conducted in English and Spanish-language review articles, published during the last 8 years on the Medline database; the key-words "ambulatory blood pressure" and "arterial hypertension" were used.

Conclusions: This auxiliary diagnostic examination has a higher predictive value for cardiovascular events than clinical room blood pressure measurement. It can improve the evaluation of cardiovascular risk in hypertensive patients. The main recommendations for its use are: refractory hypertension approach; patients with previous cardiovascular events or target organ lesions; white coat hypertension and masked hypertension diagnostics; abnormal blood pressure circadian rhythm identification, more frequent in elderly people and accountable for the aggravation of their prognosis; evaluation of diabetic patients and of pregnant women with high blood pressure values.

Keywords: Hypertension; White Coat Hypertension; Masked Hypertension; ABPM; Blood Pressure Evaluated in the Clinical Room; Primary Healthcare; Cardiovascular Disease. 